\title{
BreastScreen-based mammography screening in women with a personal history of breast cancer, Western Australian study
}

\section{Nehmat Houssami MB BS, FAFPHM, PhD, Principal Research Fellow and Associate Professor (Research) $^{1}$}

Janette J Tresham BSc(Agric), Data Manager ${ }^{2}$

Lin Fritschi MB BS, FAFPHM, PhD Professor

Liz E Wylie MB BS, FRANZCR Clinical Director, ${ }^{2}$ and Clinical Associat Professor

1 Screening and Test Evaluation Program, Schoo of Public Health, University of Sydney, Sydney, NSW.

2 BreastScreen WA

Department of Health Perth, WA.

3 Western Australia Institute for Medical Research, University of Western Australia, Perth

Royal Perth Hospita Radiology Department Radiology Department,
University of Western Australia, Perth, WA.

nehmath@

med.usyd.edu.au

MJA 2011; 195: 460-464: doi: 10.5694/mjall.10702

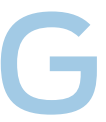

lobally, breast cancer accounts for $23 \%$ of all cancers occurring in women. ${ }^{1}$ Due to the excellent prognosis and longer life expectancy in early-stage breast cancer, the number of women with a personal history of breast cancer (PHBC) — "breast cancer survivors" - is increasing. Women with PHBC have a long-term increased risk of developing a recurrent or new cancer in the previously affected breast (where treated with breast conservation) or a contralateral cancer. ${ }^{2-4}$ Given that population mammography screening reduces breast cancer deaths, ${ }^{5}$ and that observational data show potential benefit from early detection of second breast cancers in PHBC women, ${ }^{6-10}$ international and Australian recommendations include mammography screening for these women. ${ }^{11-16}$ Annual breast clinical examination is also advised for PHBC women. ${ }^{11,13-16}$

Evidence reviews have highlighted the lack of high-quality screening evaluations and the paucity of data from screening programs for PHBC women. ${ }^{17,18} \mathrm{~A}$ recent report of outcomes from screening PHBC women who attended Breast Cancer Surveillance Consortium-affiliated mammography facilities in the United States $^{2}$ has provided international benchmarks for screening performance measures in PHBC women. Screening PHBC women is particularly relevant in Australia, where there is inconsistent policy on whether these women may access mammography through BreastScreen Australia services and where there is little evidence to inform practice.

Here, we report the first Australian study of mammography screening outcomes in women with PHBC who participated in population-based

\begin{abstract}
Objective: To evaluate mammography screening outcomes in women with a personal history of breast cancer (PHBC), who have an increased risk of recurrent or new breast cancer, relative to women without PHBC.

Design, setting and participants: Retrospective study of 713191 screening mammograms from two groups of women - those with versus those without $\mathrm{PHBC}$ - who participated in the BreastScreen WA program in Western Australia between 1997 and 2006.
\end{abstract}

Main outcome measures: Cancer detection rate (CDR), recall to assessment rate, recall positive predictive value (PPV) for cancer, and distribution of cancer characteristics within and between the two groups.

Results: Screening detected 4125 breast cancers: CDR per 10000 screens was significantly higher in women with PHBC (95.5; 95\% CI, 78.3-112.7) than in women without PHBC $(57.2 ; 95 \% \mathrm{Cl}, 55.4-58.9)$. Recall to assessment rate per 10000 screens was lower in women with PHBC $(385.2 ; 95 \% \mathrm{Cl}, 350.6-419.8)$ than in women without PHBC (504.9; 95\% Cl, 499.7-510.2). Recall PPV was higher for women with PHBC (24.8\%; $95 \% \mathrm{Cl}, 21.0 \%-28.9 \%)$ than those without PHBC (11.2\%; 95\% Cl, 10.9\%-11.6\%). Cancer characteristics were consistent with early detection (most were smaller than $2 \mathrm{~cm}$ and nodenegative) and were similarly distributed in both groups, except for tumour grade, with PHBC women having fewer low-grade cancers and slightly more highgrade cancers than women without PHBC.

Conclusions: The relative rate of cancer detection between women with $\mathrm{PHBC}$ and women without PHBC who attended an Australian population-based breast screening program was similar to estimates from international studies. Recall rates were within national standards. Screen-detected cancers had similar characteristics in both groups, except for tumour grade. These data support national integration of mammography screening for women with PHBC into BreastScreen, although evaluation of interval cancers will be necessary.

screening through the BreastScreen program. We aimed to examine rates of cancer detection and recall to assessment and to define the characteristics of screen-detected cancers in PHBC women, and to compare these outcome measures with those in concurrent screening participants who did not have PHBC.

\section{Methods}

\section{Study design and participants}

This was a retrospective study of two groups of women who participated in screening through BreastScreen WA, the Western Australian component of BreastScreen Australia, between Jan- uary 1997 and December 2006. BreastScreen WA provides free population-based mammography screening to all women aged 40 years or older, ${ }^{19}$ actively targeting women aged 50-69 years according to BreastScreen Australia policy. ${ }^{20}$ BreastScreen WA has granted PHBC women access to breast screening since its statewide implementation in 1995.

We included all screening mammograms for women who reported a PHBC. As a comparison group, we included screens for women who had screening during the same time frame and who did not report a PHBC. We used self-reported history of breast cancer (from information routinely collected at each screen) to classify the 
1 Breast cancer detection rates in women with and without a personal history of breast cancer (PHBC) participating in the BreastScreen WA program, 1997-2006

Women with PHBC

\begin{tabular}{llll}
\cline { 2 - 4 } Age group & $\begin{array}{c}\text { No. of screening } \\
\text { mammograms }\end{array}$ & $\begin{array}{c}\text { No. of } \\
\text { cancers }\end{array}$ & $\begin{array}{c}\text { Cancer detection rate per } \\
10000 \text { screens }(95 \% \mathrm{Cl})\end{array}$
\end{tabular}

Women without PHBC

$\begin{array}{ccc}\begin{array}{c}\text { No. of screening } \\ \text { mammograms }\end{array} & \begin{array}{c}\text { No. of } \\ \text { cancers }\end{array} & \begin{array}{c}\text { Cancer detection rate per } \\ 10000 \text { screens }(95 \% \mathrm{Cl})\end{array}\end{array}$

\begin{tabular}{|c|c|c|c|c|c|c|}
\hline \multicolumn{7}{|c|}{ Initial screening examinations (prevalent screening) } \\
\hline $40-49$ years & 161 & 0 & - & 57978 & 219 & $37.8(32.8-42.8)$ \\
\hline $50-69$ years & 767 & 11 & $143.4(58.6-228.2)$ & 71920 & 590 & $82.0(75.4-88.7)$ \\
\hline$\geqslant 70$ years & 263 & 4 & $152.1(42.0-385.0)$ & 5174 & 102 & $197.1(158.9-235.4)$ \\
\hline All age groups & 1191 & 15 & $125.9(62.2-158.5)$ & 135072 & 911 & $67.4(63.1-71.8)$ \\
\hline \multicolumn{7}{|c|}{ Repeat screening examinations (incident screening) } \\
\hline $40-49$ years & 423 & 6 & $141.8(52.0-306.0)$ & 68874 & 183 & $26.6(22.7-30.4)$ \\
\hline $50-69$ years & 8634 & 79 & $91.5(71.3-111.7)$ & 462646 & 2585 & $55.9(53.7-58.0)$ \\
\hline$\geqslant 70$ years & 2110 & 18 & $85.3(45.9-124.7)$ & 34241 & 328 & $95.8(85.4-106.2)$ \\
\hline All age groups & 11167 & 103 & $92.2(74.4-110.0)$ & 565761 & 3096 & $54.7(52.8-56.7)$ \\
\hline \multicolumn{7}{|c|}{ All screening examinations } \\
\hline $40-49$ years & 584 & 6 & $102.7(38.0-222.0)$ & 126852 & 402 & $31.7(28.6-34.8)$ \\
\hline $50-69$ years & 9401 & 90 & $95.7(76.0-115.5)$ & 534566 & 3175 & $59.4(57.3-61.5)$ \\
\hline$\geqslant 70$ years & 2373 & 22 & $92.7(54.0-131.5)$ & 39415 & 430 & $109.1(98.8-119.4)$ \\
\hline All age groups & 12358 & 118 & $95.5(78.3-112.7)$ & 700833 & 4007 & $57.2(55.4-58.9)$ \\
\hline
\end{tabular}

group of women with $\mathrm{PHBC}$, because this has been shown to be highly accurate. $^{21}$ Self-reported history of breast surgery (including site/side of scars) is routinely verified by the technologist at time of screening.

\section{The screening process}

BreastScreen WA participants receive two-view mammography of each breast, and all screens are read by two radiologists. Women who have a possible abnormality on mammography are recalled to assessment (this may include further imaging or needle biopsy); disagreement about recall to assessment is resolved by arbitration from a third reader. Most women recalled to assessment will be reassured that they do not have breast cancer after further testing.

Data are routinely collected from each woman who attends screening with BreastScreen WA. Participants are asked to complete a standard questionnaire (collecting demographic and breast history data), and to provide written consent for de-identified data about each screening episode to be used for program evaluation and research publication. Ethics approval was therefore not required for this study. Information from pathology and radiology or clinical reports for each screening episode is linked to client identification to ascertain outcomes in all screened women, including those referred for surgery, and to classify cancer characteristics in screening participants. These data are securely stored in dedicated BreastScreen WA databases and are routinely used to monitor quality as part of BreastScreen national accreditation processes.

\section{Outcome measures and statistical analysis}

The number of screens (initial or repeat) and the number of breast cancers detected were calculated for each group: overall, and by three age groups conventionally reported in screening participants (40-49, 50-69 and $\geqslant 70$ years). We also compared 5year age-group distributions between the two groups. We calculated cancer detection rate (CDR) per 10000 screens (overall and by age group) for initial screens (ie, the first recorded screen in the program), repeat screens, and all screens, assuming independence of observations. Recall (to assessment) rates, positive predictive value (PPV) for recall, and the proportion of screens (of those that detected cancer) that required multiple reads were calculated. Exact 95\% confidence intervals were calculated for rates and proportions. ${ }^{22}$ The distribution of cancer characteristics (histological type, size, grade and node status) was compared for the two groups using contingency tables and the $\chi^{2}$ statistic.

\section{Results}

During the study period, there were 713191 screens: 12358 in PHBC women and 700833 in women without PHBC (Box 1). More than threequarters of all screens were in women aged 50-69 years (the program's target age group). However, 5-year agegroup distributions differed between the two groups $(P<0.001 ;$ Box 2$)$. There were relatively fewer screens in the 40-49-years age group for women with PHBC (5\%) than those without $(18 \%)$, and conversely more screens in the $\geqslant 70$-years age group for PHBC women relative to those without PHBC.

Among all screening participants, 4125 breast cancers were detected. Screening detected 118 breast cancers

\section{Five-year age-group distribution* for screening examinations in women with and without a personal history of breast cancer (PHBC)}

\begin{tabular}{lcc} 
Age group & Women with PHBC & Women without PHBC \\
\hline $40-44$ years & $0.90 \%$ & $5.85 \%$ \\
$45-49$ years & $3.98 \%$ & $12.33 \%$ \\
$50-54$ years & $13.42 \%$ & $24.38 \%$ \\
$55-59$ years & $20.41 \%$ & $21.30 \%$ \\
$60-64$ years & $22.04 \%$ & $17.12 \%$ \\
$65-69$ years & $22.81 \%$ & $13.81 \%$ \\
$70-74$ years & $10.65 \%$ & $3.71 \%$ \\
$\geqslant 75$ years & $5.79 \%$ & $1.50 \%$ \\
\hline
\end{tabular}

* $\chi^{2}$ statistic for comparison of 5-year age-group distributions between the two groups, $P<0.001$. 
3 Rates of recall to assessment in women with and without a personal history of breast cancer (PHBC) participating in the BreastScreen WA program, 1997-2006

\begin{tabular}{|c|c|c|c|c|}
\hline \multirow[b]{2}{*}{ Age group } & \multicolumn{2}{|r|}{ Women with PHBC } & \multicolumn{2}{|r|}{ Women without PHBC } \\
\hline & No. & Rate per 10000 screens $(95 \% \mathrm{Cl})$ & No. & Rate per 10000 screens $(95 \% \mathrm{Cl})$ \\
\hline \multicolumn{5}{|c|}{ Initial screening examinations (prevalent screening) } \\
\hline $40-49$ years & 9 & $559.0(259.0-1035.0)$ & 6384 & 1101.1 (1074.1-1128.1) \\
\hline $50-69$ years & 51 & $664.9(482.4-847.4)$ & 7182 & $998.6(975.5-1021.7)$ \\
\hline$\geqslant 70$ years & 20 & $760.5(427.2-1093.7)$ & 481 & $929.6(846.4-997.3)$ \\
\hline All age groups & 80 & $671.7(524.5-818.9)$ & 14047 & $1040.0(1022.8-1054.0)$ \\
\hline \multicolumn{5}{|c|}{ Repeat screening examinations (incident screening) } \\
\hline $40-49$ years & 24 & $567.4(340.4-794.4)$ & 3508 & $509.3(492.5-526.2)$ \\
\hline $50-69$ years & 303 & $350.9(268.9-390.5)$ & 16600 & $358.8(353.4-364.3)$ \\
\hline$\geqslant 70$ years & 69 & $327.0(249.9-404.2)$ & 1232 & $359.8(339.7-379.9)$ \\
\hline All age groups & 396 & $354.6(319.7-389.5)$ & 21340 & $377.2(372.1-382.2)$ \\
\hline \multicolumn{5}{|c|}{ All screening examinations } \\
\hline $40-49$ years & 33 & 565.1 (372.3-757.9) & 9892 & 779.8 (764.4-795.2) \\
\hline $50-69$ years & 354 & $376.6(337.3-415.8)$ & 23782 & $444.9(439.2-450.5)$ \\
\hline$\geqslant 70$ years & 89 & $375.1(297.1-453.0)$ & 1713 & $434.6(414.0-455.2)$ \\
\hline All age groups & 476 & $385.2(350.6-419.8)$ & 35387 & $504.9(499.7-510.2)$ \\
\hline
\end{tabular}

in PHBC women, with a CDR of 95.5/ 10000 screens (95\% CI, 78.3-112.7); this was significantly higher than the

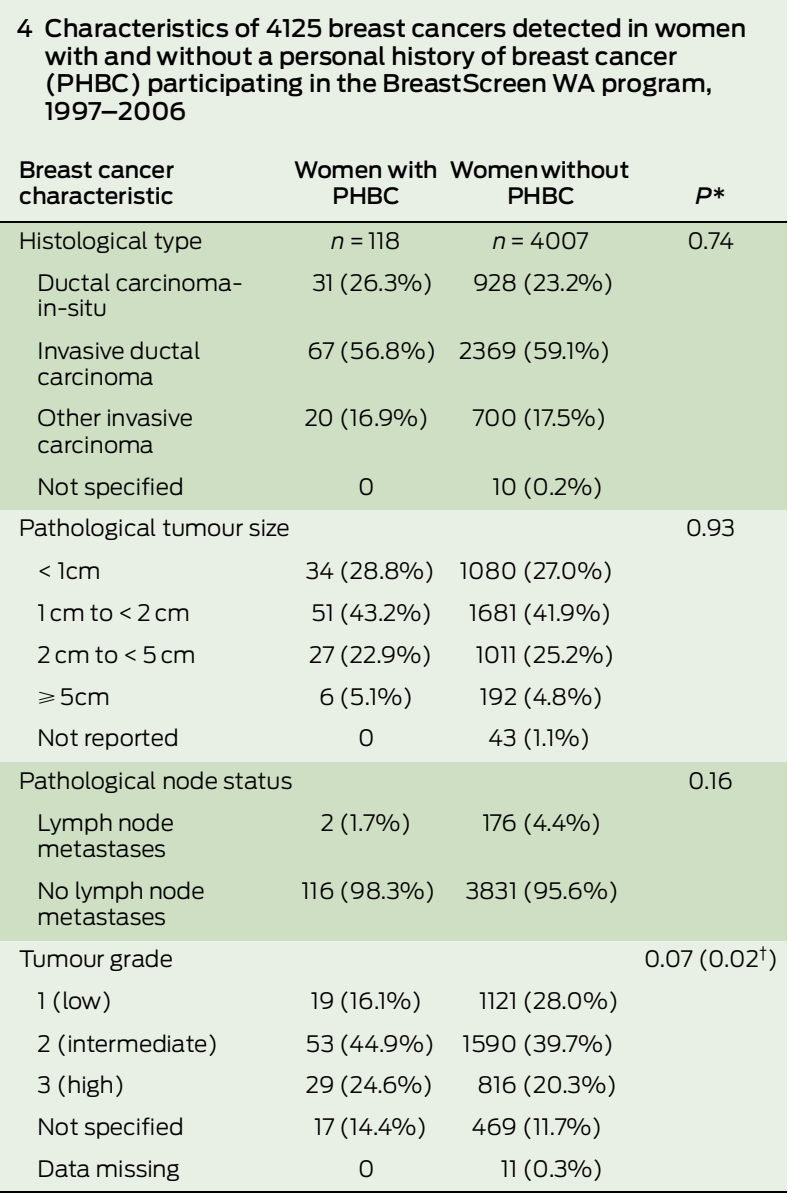

$* P$ for Pearson $\chi^{2}$ statistic for comparison of distributions. $\dagger P$ for $\chi^{2}$ statistic excluding data for not specified and missing grades.
CDR of 57.2/10000 screens (95\% CI, 55.4-58.9) for women without PHBC, in whom 4007 cancers were detected. A relatively higher $\mathrm{CDR}$ in $\mathrm{PHBC}$ women was evident in the 40-49years and 50-69-years age groups, but not in women aged $\geqslant 70$ years (Box 1).

Box 3 shows recall rates for each group: these were generally lower in PHBC women than women without PHBC (or similar in both groups) across all age groups and screening rounds. Overall, recall rates were significantly lower in PHBC women (385.2/10 000 screens; 95\% CI, 350.6419.8) than in women without PHBC (504.9/10 000 screens; 95\% CI, 499.7510.2)

PPV for recall was significantly higher for PHBC women (24.8\%; 95\% CI, 21.0\%-28.9\%) than for women without PHBC (11.2\%; 95\% CI, $10.9 \%-11.6 \%)$. In screening examinations that detected cancer, the rate of screens requiring three or more reads was 36.4/10 000 screens (95\% CI, 25.8-47.1) in the PHBC group, higher than the 15.5/10 000 screens (95\% CI, 14.6-16.4) in women without PHBC.

Box 4 summarises the characteristics of the 4125 breast cancers detected in all screening participants, by group. The distributions of tumour characteristics were not significantly different between the two groups, except for tumour grade where there was evidence $(P=0.02)$ of different distributions (PHBC women had fewer low-grade cancers and more intermediate or high-grade cancers) after excluding data for reports with unknown grade.

\section{Discussion}

We found that mammography screening through the BreastScreen WA program was associated with a higher detection rate of breast cancer for women with PHBC than for women without PHBC (95.5 v 57.2/10000 screens). Screening recall rates were within national standards, with $\leqslant 5 \%$ of all screening examinations resulting in recall to assessment for each group. $^{20}$ This is the first Australian evaluation of screening outcomes in PHBC women from a population screening program, and the second study ever to have also integrated a comparison group of women without PHBC who underwent mammography in the same screening services and time frame. The strength of our study design is that it provides an understanding of the relative screening outcomes in the two groups of women, and allows interpretation of our findings in the context of national standards for population screening. ${ }^{20}$

CDR and characteristics of detected cancers are routinely used to monitor screening outcomes and as surrogate measures of potential screening efficacy. ${ }^{15,20}$ A relatively 
higher CDR in PHBC women would be expected, as PHBC women have increased underlying risk for breast cancer, ${ }^{2-4,14}$ and also because they are likely to have had more frequent screening (mostly annual) than women without PHBC (generally biennial). The age distribution (the PHBC group had relatively more screens from older women) may have also contributed to the observed higher CDR in PHBC women, although the majority of screens in both groups were in 50-69-year-olds, the program's target age group. To interpret our findings, given the absence of standards for screening outcomes in PHBC women, we can compare our estimates with those recently benchmarked by the Breast Cancer Surveillance Consortium (BCSC), which also compared two groups by PHBC status. ${ }^{2}$ Although detection rates for each group in our study were generally higher than those reported by the $\mathrm{BCSC}$, the relative detection between groups was similar in both studies, with a 1.7-fold higher rate of cancers detected in PHBC women relative to those without PHBC in our study, and a 1.6-fold higher rate reported from the BCSC. ${ }^{2}$ Our CDRs for the group without PHBC (Box 1) demonstrate that cancer detection measures were well within BreastScreen national standards (counting in-situ and invasive cancer, CDR standards are $\geqslant 62 / 10000$ for prevalent screens and $\geqslant 42 / 10000$ for incident screens). ${ }^{20}$

Recall rates allow monitoring of the burden (potential harm) of screening from unnecessary testing. ${ }^{5,20}$ Our study reports the first international estimates of screening recall rates in PHBC women from a populationbased mammography program. We found that recall rates were lower in PHBC women than in women without PHBC (385.2 v 504.9/10 000 screens), whereas recall PPV (for cancer detection) was relatively higher in PHBC women, due to both fewer recalls and a relatively higher CDR in PHBC women. Because there are no published recall data for PHBC women who participated in population-based screening, we cannot compare our recall estimates to other studies. The BCSC study did not estimate recall rates but reported that
PHBC women were relatively more likely to require additional imaging. ${ }^{2}$ A possible explanation for the lower recall in our PHBC group may be the screen-reading strategy used in the program - although more screens in PHBC women required three or more reads, these reads effectively arbitrated such cases. It may also be (partly) due to having relatively more screens from older women in the PHBC group.

Our study has shown that mammography screening detected second breast cancers in PHBC women that were predominantly smaller than $2 \mathrm{~cm}$ and node-negative (Box 4). The characteristics of cancers detected in PHBC women were generally consistent with early-detected cancers, and, except for tumour grade, were similarly distributed to those of cancers detected in women without PHBC. Tumour grade distribution differed significantly between the two groups, with PHBC women having fewer lowgrade cancers and slightly more highgrade cancers relative to women without PHBC. This may reflect underlying host factors in PHBC women, possibly genetic or biological determinants, or may be a manifestation of tumour biology "selection" in a group of women who are likely to have received systemic therapy for their first breast cancer.

Our findings are relevant and timely for breast screening practice in Australia, where there is inconsistent policy on screening PHBC women (partly due to paucity of data from screening programs), and given that an evaluation of BreastScreen has recommended consideration of national provision of screening for PHBC women through BreastScreen services. ${ }^{15}$ Although our study represents the first Australian report of screening in PHBC women, our results should be considered with awareness of some study limitations. As we did not have data on interval cancers, we were unable to estimate screening sensitivity, and we therefore cannot make recommendations on the time frame (after first cancer diagnosis) at which PHBC women might be offered entry into BreastScreen. The BreastScreen evaluation has recommended annual screening for PHBC women from 5 years after first cancer diagnosis, ${ }^{15}$ and this seems reasonable based on the recent evidence. $^{2}$

We were unable to examine screening outcomes according to the first cancer treatment received for PHBC women. Characterising this aspect of screening outcomes in PHBC women should be examined in future evaluations because the detection capability of mammography is modified by treatment received for the first cancer. ${ }^{2}$ Although ours is one of the largest studies of breast screening in this context, we had modest numbers of second cancers for age-group and screening-round strata in the PHBC group, leading to some strata-specific wide confidence intervals. Therefore, we have focused on the precise estimates for all screens in defining our conclusions. Also, because some women had several screens, confidence intervals around our estimates may have been slightly wider if we had allowed for clustering in women with repeat screens. A further potential limitation is that screening outcomes for PHBC women in BreastScreen WA may not be generalisable to women undergoing screening in the private sector (where there are few available data on screening participants and outcomes).

Mammography screening in PHBC women who participated in BreastScreen WA was associated with a higher CDR and lower recall rates relative to women without PHBC. Cancer characteristics were predominantly consistent with early detection in PHBC women and were similar to characteristics of cancers detected in women without PHBC, with the exception of tumour grade. Our findings support the role of mammography screening for PHBC women, and support allowing these women (in target age groups for screening) to have nationally consistent access to mammography screening through BreastScreen. Future research into screening $\mathrm{PHBC}$ women requires both epidemiological and clinical (radiological) evaluation of interval cancers - this should be planned carefully and early, if implementation of a national policy allowing PHBC women access to BreastScreen is adopted. 
Acknowledgements: Nehmat Houssami receives funding through National Health and Medical Research Council program grant 633003 to the Screening and Test Evaluation Program.

Competing interests: No relevant disclosures.

Received 4 Jun 2011, accepted 29 Aug 2011.

1 Jemal A, Bray F, Center MM, et al. Global cance statistics. CA Cancer J Clin 2011; 61: 69-90.

2 Houssami N, Abraham LA, Miglioretti DL, et al. Accuracy and outcomes of screening mammography in women with a personal history of early-stage breast cancer. JAMA 2011; 305 : 790-799.

3 Buist DS, Abraham LA, Barlow WE, et al Diagnosis of second breast cancer events after initial diagnosis of early stage breast cancer. Breast Cancer Res Treat 2010; 124: 863-873. [Epub 2010 Aug 11.]

4 Punglia RS, Hassett MJ. Using lifetime risk estimates to recommend magnetic resonance imaging screening for breast cancer survivors. J Clin Oncol 2010; 28: 4108-4110.

5 Glasziou P, Houssami N. The evidence base for breast cancer screening. Prev Med 2011; 53 . 100-102. doi: 10.1016/j.ypmed.2011.05.011.

6 Lu WL, Jansen L, Post WJ, et al. Impact on survival of early detection of isolated breast recurrences after the primary treatment for breast cancer: a meta-analysis. Breast Cancer Res Treat 2009; 114 403-412.

7 Houssami N, Ciatto S, Martinelli F, et al. Early detection of second breast cancers improves prognosis in breast cancer survivors. Ann Oncol 2009: 20: 1505-1510.

8 Lash TL, Fox MP, Buist DS, et al. Mammography surveillance and mortality in older breast cancer survivors. J Clin Oncol 2007; 25: 3001-3006.

9 Ciatto S, Miccinesi G, Zappa M. Prognostic impact of the early detection of metachronous contralateral breast cancer. [Erratum in Eur J Cancer 2005; 41: 477.] Eur J Cancer 2004; 40: 1496-1501.

10 Lu W, Schaapveld M, Jansen L, et al. The value of surveillance mammography of the contralateral breast in patients with a history of breast cancer. Eur J Cancer 2009; 45:3000-3007.

11 Khatcheressian JL, Wolff AC, Smith TJ, et al. American Society of Clinical Oncology 2006 update of the breast cancer follow-up and management guidelines in the adjuvant setting. $J$ Clin Oncol 2006; 24: 5091-5097.

12 Lee CH, Dershaw DD, Kopans D, et al. Breast cancer screening with imaging: recommendations from the Society of Breast Imaging and the ACR on the use of mammography, breast MRI, breast ultrasound and other technologies for the detection of clinically occult breast cancer. J Am Coll Radiol 2010; 7: 18-27.

13 Carlson RW, Allred DC, Anderson BO, et al. Breast cancer. Clinical practice guidelines in oncology. Natl Compr Canc Netw 2009; 7: 122-192.

14 Hayes DF. Clinical practice. Follow-up of patients with early breast cancer. N Engl J Med 2007; 356: 2505-2513.
15 BreastScreen Australia Evaluation Advisory Committee. BreastScreen Australia Evaluation evaluation final report. Screening monograph no. 1/2009. Canberra: Australian Government Department of Health and Ageing, 2009.

16 Brennan ME, Houssami N. Overview of long term care of breast cancer survivors. Maturitas 2011; 69: 106-112.

17 Grunfeld E, Noorani H, McGahan L, et al. Surveillance mammography after treatment of primary breast cancer: a systematic review. Breast 2002; 11: 228-235.

18 Houssami N, Ciatto S. Mammographic surveillance in women with a personal history of breast cancer: how accurate? How effective? Breast 2010; 19: 439-445.

19 Department of Health, Government of Western Australia. BreastScreen WA [website]. http:// www.breastscreen.health.wa.gov.au (accessed Sep 2011).

20 Australian Institute of Health and Welfare. BreastScreen Australia monitoring report 20052006. Canberra: AlHW, 2009. (AlHW Cat. No. CAN 44.)

21 Abraham L, Geller BM, Yankaskas BC, et al. Accuracy of self-reported breast cancer among women undergoing mammography. Breast Cancer Res Treat 2009; 118: 583-592.

22 Gardner MJ, Altman DG, editors. Statistics with confidence. London: British Medical Journal, 1989. 